\title{
Vertex Quasiprimitive and 2-Arc Transitive Graphs of Order $2 p^{\mathrm{e}}$
}

\author{
Li Ma
}

School of Mathematics and statistics, Qujing Normal University, Yunnan, Qujing, 655011, China 821962782@qq.com

Keywords: 2-arc tansitive graph, Quasiprimitive permutation group, O'Nan-Scott type.

Abstract: A graph $\Gamma$ is called 2-arc transitive graph if its automorphism group $\operatorname{Aut}(\Gamma)$ is transitive on the set of 2-arcs. In this paper, we main classfy the vertex quasiprimitive 2-arc transitive graphs of order $2 \mathrm{p}^{\mathrm{e}}$

\section{Introduction}

Let $\Gamma=(\mathrm{V}, \mathrm{E})$ be a graph with vertex set $\mathrm{V}$, edge set $\mathrm{E}$. For $X \leq \operatorname{Aut}(\Gamma)$, a group of automorphisms. $\Gamma$ is called G-vertex transitive or G-edge transitive if $\mathrm{G}$ is transitive on $\mathrm{V}$ or $\mathrm{E}$, respectively. The motivation for this paper came from a study of $s$-arc transitive graphs, $s \geq 2$. An s-arc, $\mathrm{s} \geq 1$, in a graph $\Gamma$ is a sequence $\alpha_{0}, \alpha_{1}, \cdots, \alpha_{s}$ of $\mathrm{s}+1$ vertices of $\Gamma$ such that, for all $1 \leq i \leq s$, $\alpha_{i+1} \neq \alpha_{i-1}$. A graph $\Gamma$ is said to be s-arc transitive if its automorphism group $\operatorname{Aut}(\Gamma)$ is transitive on the set of the s-arcs, and a subgroup $G$ of $\operatorname{Aut}(\Gamma)$ is said to be s-arc transitive on $\Gamma$ if $G$ is transitive on the set of s-arcs of $\Gamma$. By[6], it is known that a finite, connected, s-arc transitive graph, $\mathrm{s} \geq 2$, which is not bipartite, is a cover of an s-arc transitive graph with a subgroup of automorphisms quasiprimitive on vertices. This paper aims to characterize undirected vertex quasiprimitive 2-arc transitive graphs of order $2 p^{e}$ with $p$ is an odd prime. Let $\mathrm{G}$ be a group, $\mathrm{H}$ be a subgroup of $G$, and $S$ be s subset of $G$. Then the coset graph of $G$ with respect to $H$ and $S$ to be the directed graph with vertex set $[\mathrm{G}: \mathrm{H}]$ and such that, for any $H x, H y \in V$. Hx is connected to Hy if and only if $y x^{-1} \in H S H$. and denote the digraph by $\operatorname{Cos}(\mathrm{G}, \mathrm{H}, \mathrm{HSH})$. By [9], For any $G \leq \operatorname{Aut}(\Gamma)$, $\Gamma$ is $(\mathrm{G}, 2)$-arc transitive if and only if $\Gamma \cong \operatorname{Cos}(G, H, H S H)$ such that $g^{2} \in H$ and $H$ acts(by coset action) 2-transitive on $\left[H: H \cap H^{g}\right]$. Let $N \triangleleft X$, and let $\bar{B}$ be the set of N-orbits in V. Then the normal quotient graph $\Gamma_{N}$ of $\Gamma$ induced by $\mathrm{N}$ is the graph with vertex set $\bar{B}$ such that $B, B_{1} \in \bar{B}$ are adjacent in $\Gamma$ to some vertex $v \in B_{1}$. If $\Gamma$ and $\Gamma_{N}$ have the same valency, then $\Gamma$ is called a normal cover of $\Gamma_{N}$. 


\section{Vertex quasiprimitive}

A permutation group $X \leq \operatorname{Sym}(\Omega)$ is called quasiprimitive if each nontrivial normal subgroups of $\mathrm{X}$ is transitive on $\Omega$. Let $\mathrm{N}=\operatorname{Soc}(\mathrm{X})$ be the socle of $\mathrm{X}$, that is the product of all minimal normal subgroups of $\mathrm{X}$. First, we give a characterization of quasiprimitive permutation groups of degree $2 p^{e}$.

Lemma 2.1: Let $X$ be a quasiprimitive permutation group on $\Omega$ of degree $2 p^{e}$, where $p$ is an odd prime. Then $\mathrm{X}$ is almost simple(AS) group or product action(PA) group.

proof : Let $\mathrm{N}$ be a minimal normal subgroup of $\mathrm{X}$. Then $N \cong T^{l}$, where $\$ \mathrm{l}$ geq $1 \$$ and $\mathrm{T}$ is a simple group. Since $\mathrm{X}$ is quasiprimitive on $\Omega, \mathrm{N}$ is transitive on $\Omega$. Then for any $\alpha \in \Omega$, we have $\left|N: N_{\alpha}\right|=|\Omega|=p^{e}$. If $\mathrm{T}$ is abelian, then $T \cong Z_{p}$, and $N \cong Z_{p^{e}}$ is regular on $\Omega$. If $\mathrm{l}=1$ and $\mathrm{N}$ is nonabelian, then $\mathrm{X}$ is an almost simple group. If $l \geq 2$, then $N=T_{1} \times \cdots T_{l}=T^{l}$ with $l \geq 2$, and $\mathrm{T}$ is a nonabelian simple group. Since $\left|N: N_{\alpha}\right|=|\Omega|=2 p^{e}$ and $\left|T_{1}:\left(T_{1}\right)_{\alpha}\right|=\left|N:\left(T_{1}\right)_{\alpha} \times \cdots T_{l}\right|$ divides $\left|N: N_{\alpha}\right|$, we

conclude that $\left(T_{1}\right)_{\alpha}$ has index p-power or double p-power in T. In particular, $\mathrm{X}$ is type of product action.

\section{Almost Simple case}

Suppose that $\mathrm{X}$ is an almost simple group, then $T \leq X \leq T$.Out $(T)$, and thus for any $\alpha \in \Omega, \quad T_{\alpha}$ has index $2 p^{e}$ in T. By [8], we have the following lemma.

Lemma 2.2: $\quad$ Let $\mathrm{T}$ be a nonabelian simple group that has a subgroup $\mathrm{H}$ or $\mathrm{K}$ of index $2 p^{e}$ with $p^{e}$ odd prime, where $\mathrm{K}$ is a maximal subgroup of $\mathrm{T}$ but $\mathrm{H}$ is not a maximal subgroup of $\mathrm{T}$. Then $\left(T, T_{\alpha}\right)$ has been listed in the table1.

where $P_{1}$ is parabolic subgroup and $|\mathrm{T}: \mathrm{H}|=|\mathrm{T}: \mathrm{K}|=\mathrm{d}$.

When $\left(T: T_{\alpha}\right)=\left(A_{6}: A_{5}\right),\left(A_{6}, 3^{2}: 4\right),\left(A_{2 p^{l}}: A_{2 p^{l}-1}\right),\left(M_{22}: L_{3}(4)\right)$ or $\left(L_{2}(4): D_{10}\right)$,

By [4], T is 2-transitive on $\left(T: T_{\alpha}\right)$. That is $\Gamma \cong K_{6}, K_{10}, K_{2 p^{l}}, K_{22}, K_{6}$, respectively.

When $(T: H)=\left(L_{2}(4), S_{3}\right)$, there exist an involution element $g \in T$ such that $H \cap H^{g}=Z_{2}$ and $\langle H, g\rangle=G$. Thus $\Gamma \cong \operatorname{Cos}(G, H . H g H)$ is Petersen graph. 
Table1

\begin{tabular}{|c|c|c|c|}
\hline $\mathrm{T}$ & K & $\mathrm{H}$ & $\mathrm{d}=2 p^{l}$ \\
\hline$A_{2 p^{l}}$ & $A_{2 p^{l}-1}$ & & $2 p^{l}$ \\
\hline$A_{6}$ & $A_{5}$ & & 6 \\
\hline$A_{6}$ & $3^{2}: 4$ & & 10 \\
\hline$M_{11}$ & & $A_{6}$ & 22 \\
\hline$M_{22}$ & $L_{3}(4)$ & & 22 \\
\hline$L_{2}(4)$ & $D_{6}$ & & 10 \\
\hline$L_{2}(4)$ & $D_{10}$ & & 6 \\
\hline$L_{n}\left(p^{f}\right)$ & $P_{1}$ & & $\frac{q^{n}-1}{q-1}$ \\
\hline$L_{n}\left(p^{f}\right)$ & & $\mathrm{H}$ & \\
\hline$L_{n}\left(p^{f}\right)$ & & $O_{p}\left(P_{1}\right) L_{1} R$ & \\
\hline$P S p(4,3)$ & & $2^{4}: D_{10}$ & $2 \cdot 3^{4}$ \\
\hline
\end{tabular}

If $(\mathrm{T}, \mathrm{K})=\left(S p_{2 m}\left(2^{f}\right),{O^{-}}^{-}{ }_{2 m}(2)\right)$ with $\mathrm{f}=1, \mathrm{~m}=2$, then $T \cong S p_{4}(2) \cong S_{6}$ is not a simple group.

If $(\mathrm{T}, \mathrm{K})=\left(S p_{2 m}\left(2^{f}\right), O^{+}{ }_{2 m}\left(2^{f}\right)\right)$ with $\mathrm{fm}=2$, when $\mathrm{f}=1, \mathrm{~m}=2, T \cong S p_{4}(2) \cong S_{6}$ is not a simple group; $\mathrm{f}=2, \mathrm{~m}=1, \quad T \cong S p_{2}(4) \cong L_{2}(4) \cong A_{5}$ and $K=O^{+}{ }_{2}(4)$

If $(T, K)=\left(\Omega^{-}{ }_{2 m}\left(2^{f}\right), S p_{2 m-2}\left(2^{f}\right)\right)$ with $\mathrm{fm}=2$, then $\mathrm{f}=1, \mathrm{~m}=2$. That is

By [8], $\Gamma$ is $(\mathrm{G}, 2)$ - arc transitive if and only if $\Gamma \cong \operatorname{Cos}(G, H, H g H)$ such that

$g^{2} \in H$ and $\mathrm{H}$ acts(by coset action) 2-transitive on $\left[H: H \cap H^{g}\right]$. Next we need consider the rest of the group on the form one by one.

Lemma 2.3: Let $\Gamma$ be a $(G, 2)$-arc transitive with vertex V. Assume that $G$ is quasiprimitive on $V$, $|\mathrm{V}|=22$, and $\operatorname{Soc}(\mathrm{G})=\mathrm{T}=M_{11}$. Then $\Gamma$ is $(\mathrm{G}, 2)$-transitive graph with order 22 , and valency 6 .

Proof: By [4], it is known that $\operatorname{Out}(\mathrm{T})=1$, since $\mathrm{G}$ is type of AS, 
$\mathrm{G}=\mathrm{T}$.Out $(\mathrm{T}) \cong \mathrm{M}_{-}\{11\}$, and $G_{\alpha}=T_{\alpha}$. Out $(\mathrm{T})=A_{6}$. There exist an element $g^{2} \in A_{6}$ such that $H \cap H^{g}=A_{5}$, then $\mathrm{T}$ is 2-transitive on $\left[H: H \cap H^{g}\right]$. Thus $\Gamma$ is $(\mathrm{G}, 2)-$

transitive graph with order 22, and valency 6.

\section{Product Action case}

Now we assume that $l \geq 2$, then $\mathrm{X}$ is type of product action and

$\mathrm{N}=T_{1} \times \cdots T_{l} \cong T^{l}$ with $l \geq 2$, and $\mathrm{T}$ is a nonabelian simple group. Since $\left|N: N_{\alpha}\right|=|\Omega|=2 p^{e}$ and $\left|T_{1}:\left(T_{1}\right)_{\alpha}\right|=\left|N:\left(T_{1}\right)_{\alpha} \times T_{2} \times \cdots T_{l}\right|=2 p^{e}$ divides $\left|N: N_{\alpha}\right|$, we

conclude that $\left(T_{1}\right)_{\alpha}$ has index p-power or double p-power in T. We only need consider the case that $\left(T_{1}\right)_{\alpha}$ has index p-power in T. In this case, By [8], $\left|T_{1}:\left(T_{1}\right)_{\alpha}\right|$ has been listed in the following table:

Table 2

\begin{tabular}{|c|c|c|}
\hline$T$ & $T_{\alpha}$ & $\left|T: T_{\alpha}\right|$ \\
$A_{p^{r}}$ & $A_{p^{r-1}}$ & $p^{r}$ \\
\hline$P S L(d, q)$ & $P_{1}$ & $\frac{q^{a}-1}{q-1}$ \\
\hline$M_{11}$ & $M_{10}$ & 11 \\
\hline$M_{23}$ & $M_{22}$ & 23 \\
\hline$P S L(2,11)$ & $A_{5}$ & 11 \\
$P S U(4,2)$ & $Z_{2}^{4}: A_{5}$ & 162 \\
\hline
\end{tabular}

Since $\left|N: N_{\alpha}\right|=|\Omega|=2 p^{e}$, and $\left|N:\left(T_{\alpha}\right)^{l}\right|=p^{r l}$, we have that $\left|N_{\alpha}:\left(T_{\alpha}\right)^{l}\right|=2$. Then one of the following holds:

(1) $\mathrm{T}=M_{11}, \quad T_{\alpha}=M_{10}$;

(2) (2)T=PSL(d,q), $T_{\alpha}=q^{d-1} \frac{1}{(d-1, q)} G L(d-1, q)$, where $(\mathrm{d}-1, \mathrm{q}-1)$ is even.

\section{References}

[1] P. Bergau and D. Garbe,Non-orientable and orientable regular maps, Lect. Notes Math. 1398, Springer , 1989, 29-42.

[2] H. R. Brahana, Regular maps and their groups, Amer. J. Math. 1927(49), 268-284.

[3] M. D. E. Conder, Hurwitz groups - a brief survey, Bull. Amer. Math. Soc. 1990(23) ,359-370.

[4] J. H. Conway, R. T. Curtis, S. P. Norton, R. A. Parker and R. A. Wilson, Atlas of Finite Groups, Clarendon Press, Oxford, 1985. 
[5] H. S. M. Coxeter and W. O. J. Moser, Generators and Relations for Discrete Groups, Springer, New York, 1980.

[6] S. F. Du, J. H. Kwak and R. Nedela, A classification of regular embeddings of graphs of order a product of two primes, J. Alg. Combin. 2004(19), 123-141.

[7] S. F. Du and F. R. Wang, Nonorientable regular embeddings of graphs of order a product of two distinct primes, in preparation.

[8] R.M.Guralnick, Subgroups of prime power index in a simple group, J.Algebra 1983, 304-311.

[9] G. A. Jones, Maps on surfaces and Galois groups, Math. Slovaca. 1997(47) 1-33.

[10]C. H. Li, JiangMin. Pan, Li. Ma, Locally primitive graphs of prime-power order. J. Aust. Math. Soc 2008, 1-12. 